\title{
KMU-Patentaktion zum Schutz von Ideen
}

Mit der Initiative SIGNO fördert das Bundesministerium für Wirtschaft und Technologie (BMWi) Unternehmen bei der Sicherung und Verwertung ihrer Ideen. Information und Unterstützung bieten hierbei die SIGNO-Netzwerkpartner.

m Land der Dichter und Denker erfolg-

ka 15.000 Gebrauchsmusteranmeldungen. „Die Zahl der schutzwürdigen Entwicklungen liegt in Deutschland jedoch deutlich höher. Es sind vor allem kleinere und mittlere Unternehmen, die den Aufwand und/oder die Kosten für Schutzrechte scheuen“, weiß Anja Dreher, Geschäftsführerin der AHE | engineering aus Memmingen. Das im Technologie-und Innovationsmanagement tätige Unternehmen ist SIGNO-Netzwerkpartner und berät und unterstützt Unternehmen im Rahmen der vom BMWi geförderten Initiative SIGNO. Mit der KMU-Patentaktion will das Ministerium die Rahmenbedingungen für Innovationen in Deutschland verbessern und so die Umsetzung von Forschungs- und Entwicklungsergebnissen in marktfähige Produkte steigern. Ziel ist, die Wettbewerbsfähigkeit von kleineren und mittleren Unternehmen zu sichern.

\section{Umfassende Unterstützung}

„Das zielgruppenspezifische Angebot von SIGNO umfasst verschiedene Aktivitäten und Fördermaßnahmen, mit denen einerseits Innovationen angeregt werden. Andererseits beinhaltet es Informationen und Unterstützung, wenn es um das Erfindungs- und Patentwesen sowie die wirtschaftliche Nutzung von Patenten geht“, erklärt Anja Dreher. Im Einzelnen handelt es sich dabei um folgende fünf Teilpakete:

1. Die Recherche zum Stand der Technik ist Voraussetzung, um die $\mathrm{Pa}-$ tentfähigkeit abschätzen zu können und eine optimale Basis für das Anmeldeverfahren zu schaffen.

2. Anhand der Kosten-Nutzen-Analyse lässt sich die wirtschaftliche Sinnhaftigkeit einer Patentanmeldung ermitteln. Sie dient darüber hinaus als Mittel, die Verwertungschancen einer Erfindung frühzeitig abzuschätzen. Statt einer Kosten-Nutzen-Analyse kann auch eine ausführliche Technologiebewertung durchgeführt werden.

3. Durch die patentanwaltliche Unterstützung bei der Patent- oder Gebrauchsmusteranmeldung für Deutschland soll vermieden wer- den, dass Anmeldungen aufgrund von Fehlern, beispielsweise durch unklare Formulierungen, zurückgewiesen werden.

4. Durch professionelle Unterstützung soll die erfolgreiche Umsetzung und wirtschaftliche Verwertung einer geschützten Erfindung verbessert werden.

5. Die Beratung und Unterstützung bei der Anmeldung von Patenten oder Gebrauchsmustern außerhalb Deutschlands hat das Ziel der erfolgreichen internationalen Vermarktung einer Erfindung.

Gefördert werden jeweils bis zu 50 Prozent der anfallenden Kosten im Rahmen von Höchstbeträgen. Das Angebot der SIGNO-Initiative wendet sich an alle KMU, die noch kein Patent angemeldet haben beziehungsweise deren letzte Anmeldung länger als fünf Jahre $z^{-}$ rückliegt.

Weitere Infos: Anja Dreher, Tel.: 08331/8333722, signo@ahe-engineering. com, www.signo-deutschland.de

\section{Neuer Leiter Klebstoffe an der ZHAW}

$D^{\prime}$ r. Christof Brändli, vorher bei Dow Europe tätig, hat die Leitung des Labors Klebstoffe und Polymere Materialien an der Zürcher Hochschule für Angewandte Wissenschaften in Winterthur (Schweiz) übernommen.

Das Labor Klebstoffe und Polymere Materialien deckt einen großen Aufgabenbereich ab: von der Synthese und Formulierung von Klebstoffen bis hin zum Testen und Analysieren von Struktur-Eigenschaftsbeziehun- gen polymerer Materialien. Der Aufgabenschwerpunkt des entsprechend ausgestatteten Labors liegt in der angewandten Forschung- und Entwicklung von thermoplastischen sowie reaktiven Klebstoffsystemen mit Industriepartnern.

Christof Brändli startete seine Klebstoffkarriere bei Dow Europe als Entwicklungsleiter für Strukturklebstoffe für den Automobilbereich und war zuletzt für das Europageschäft zuständig.

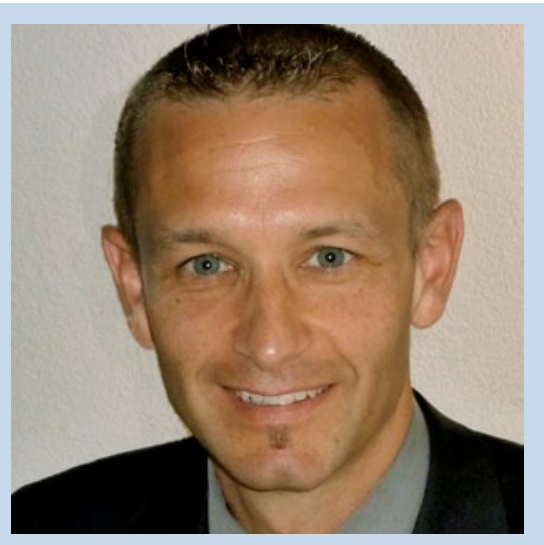

Dr. Christof Brändli, neuer Leiter des Labors Klebstoffe und Polymere Materialien an der Zürcher Hochschule für Angewandte Wissenschaften in Winterthur. 